INTERNATIONAL JOURNAL OF MULTIDISCIPLINARY RESEARCH AND ANALySis

ISSN(print): 2643-9840, ISSN(online): 2643-9875

Volume 05 Issue 02 February 2022

DOI: 10.47191/ijmra/v5-i2-07, Impact Factor: 6.072

Page No.- 287-293

\title{
Learning of Knowledge Graphs with Entity Descriptions
}

\author{
Yacouba Conde ${ }^{1}$, Zhoulianying ${ }^{2}$ \\ 1,2Jiangsu University, 301 Xuefu Road, Jingkou, Zhenjiang, Jiangsu, 212013, P.R. China
}

\begin{abstract}
The representing learning makes specialty of knowledge graph and it indicates the difference between different entities. The knowledge graph representing with the low-dimensional space. In fact, most of the method usually concern of description of entity which is hard for existing strategies to take benefit of. Here, we recommend a new representing learning method with knowledge graphs that uses entity description. We evaluate our method on two tasks like knowledge graph and entity classification. Experimental effects on actual-world datasets show that our version plays higher than different baseline fashions, especially under the zero-short setting, which indicate that our technique for novel the entity description.
\end{abstract}

\section{INTRODUCTION}

The indication of the Knowledge graph technique is based information including web search, question answering, and understanding graphs (KG) could not be imagined without them the ideal expertise graph generally represents understanding as multi-relational facts, and additional triples which is referent to as the entities (Jia et al., 2022). The primary effects are based on the symbolic identity of the people advocate with different kind of diverse kinds of knowledge graph(Bellomarini et al., 2022; Z. Li et al., 2021).

The length of Knowledge graph strategical representing the infeasible on the big KG due to the computational inefficiency and sparse dataset. The trouble of this is resolve by the illustration of representing the learning graph for KG which has been proposed to KG and it including each entity with knowledge graph inference and acquisition(Pham et al., 2022).

In parent, we display the descriptions of entities in a fact triple taken with rich semantic information about the entities. The vital base of understanding the base maintained with different dialogues and attitudes(Shao et al., 2021). Likewise, we indicate the few descriptions with two entities in fact of triple sampled from the entitle by web.

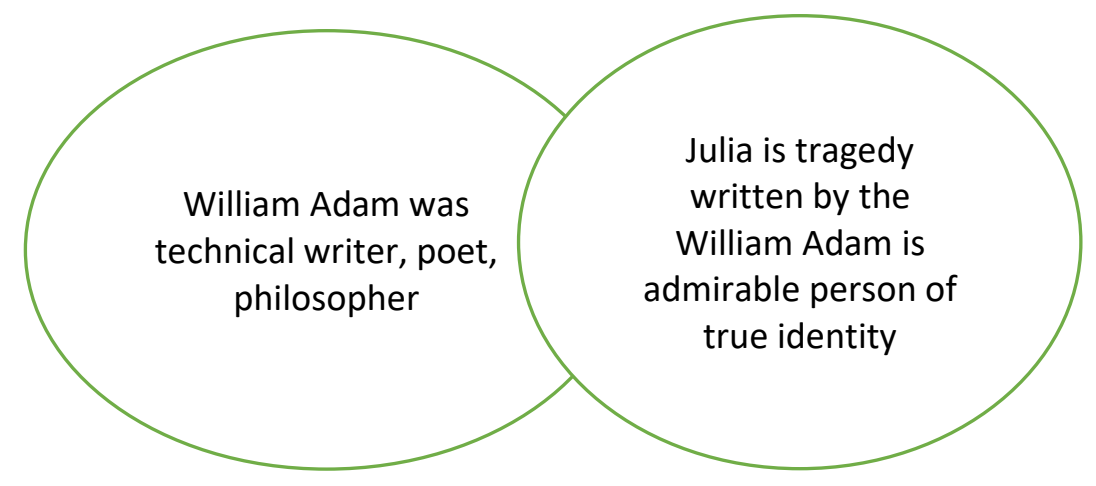

Figure 1: Entity description by web

In this case, the based information is conducted by web. The William Adam character shows the writer, poet and philosopher, Julia determined his personality as an admirable and true identity(J. Li et al., 2022). For instance, in this description the basic strategies aren't always trivial for the knowledge graph and representing the learning graph but the shows the strategies to make use the entity instance(C. Li et al., 2021).

In the description embodied knowledge representing learning (KDAL(CBOW)) model the indicated test is responsible for the both modeling the corresponding fact of triples and description of model. The embedded ding of business entity is liable for each 


\section{Learning of Knowledge Graphs with Entity Descriptions}

making the proper version triple and making the model of its definition. In reality threefold(Tiddi \& Schlobach, 2022), we comply with the standard RL technique. The look at 3-dimensional relation as a translation from the top to the tail commercial enterprise with different entity. In this manner, initiative embedding and relationships are studied to growth the possibilities of this translation(Yu et al., 2021). At that point of research implications, within the busines case, we are able to additional identify to boom the variety and possible probabilities of predicting its meaning. In this instance, we conducted two codes to represent the semantics of term of business, including a rapid continuous bag-of-words and deep version of structure. Comparatively, version assumes the phrase order that is stress structure of phrases inside the sentence context which is consider for the application of bag-of-words (Bellomarini et al., 2022).

We analysis the co-effectiveness of the description embodied knowledge representing learning in two different tasks, which is consisted of the computation of the graph and enterprise classifications. The descriptive results shows that the description embodied knowledge representing learning model is surpasses the lawsuits of the artificial intelligence for those features, especially we consider the scenario of bit zero shot, where the knowledge graph existence the representing the learning strategies in the novel entities (Wang, Hao, et al., 2020; Zhao et al., 2020). In this case the existing representing of learning methods of knowledge graph don't realize those novel entities, as no embedding have been learning in them. However, the description embodied knowledge representing learning can routinely structure of these novel entities of the descriptions. The assessment of 0 -shot settings displays that description embodied knowledge representing learning model, which can nonetheless get better effectiveness of different capabilities (Q. Li et al., 2020). This demanding structure demonstrates the brilliant integration and durability of description embodied knowledge representing learning model, that is crucial to the huge knowledge graph scale and its overall performance of the website (Zhao et al., 2021).

\section{WORK ORIENTATION}

The work orientation has been distributed in the variety of ramification of ways to model facts this is carefully associated with statistics graphs, a lot of which encode each commercial enterprise and relationships in a low-dimensional low vector continuous velocity(Huang et al., 2021). The relationship capabilities among the key companies and with a low-vector space determined as

$$
E(h a, r, t e)=\|h a+r-t e\|
$$

In the Eq 1 the indicator represent the translates relationships as translation capabilities among key companies and the tail within the space of a low-vector vector. The power function is described as $E(E(h a, r, t e)=\|h a+r-t e\| . . . .$. indicating that the tail should be nearest neighbor of $h a+r$. The E properly is indicated one to one intersection as the same time as having troubles modeling relationships from the model relationship and exposing $h a$ and te to hyperplane precise relationships vessels(Bai et al., 2021). It's a version of different type of the organizations and in term relationship in one of kind semantic business and surroundings projects from the enterprise area to the relationship space while to solve the E problem by description of model and as hyperplanes and exposing $h a$ and te a kind semantic relationships and business projects with proposes a consultant mastering model based totally on multiple steps in phrases of approach (Lampropoulos et al., 2020).

Many present RL-primarily based translation strategies for KGs awareness best on structural understanding between organizations, regardless of which rich coded information in business descriptions. moreover, because of the restrict on the representation of the business representation, these fashions also cannot assure three times if as a minimum one enterprise is outside the KG. However, this case may be dealt with thru our KDAL (CBOW) model. There are several approaches to use textual statistics to help examine KG illustration. (Socher et al. 2013) pro represents NTN and represents the commercial enterprise as a widespread for the embedding of its names at the enterprise name, allowing for the sharing of textual facts determined in comparable commercial enterprise names. (Wang et al. 2014a) combines business embedding and word embedding in a continuous vector technique with alignment modeling models the usage of Wikipedia business names or anchors. (Zhong et al. 2015) expands the incorporated version and aligns facts and textual content embedding with business definitions. these two capabilities constitute new agencies using the embedding of related commercial enterprise names(Tiwari et al., 2021). Represents companies with commercial enterprise names or a fee of word embedding in descriptions. however, their use of definitions ignores the phrase or definitions, and the use of company names is elaborate and ambiguous (Ko et al., 2021). Moreover, in the case of a easy, insignificant case, the embedding of recent commercial enterprise names is regularly missing in education information. Our model can at once create presentations from descriptions to avoid such troubles, now not just to use commercial enterprise descriptions as extra statistics. 


\section{Learning of Knowledge Graphs with Entity Descriptions}

\section{PROBLEM FORMULATION}

We first introduce the texts used in this paper. when given three $h a, r, t e \in \mathrm{G}$ while $h a, r, t e \in \mathrm{E}$ represents agencies and $r \in \mathrm{R}$ represents relationships (Shi et al., 2020). E is the commercial enterprise set and $R$ is the relationship set. $G$ stands for schooling set. Every enterprise and related embassy take prices in $R_{k}$

Description The building structure represent primarily based presentations of the $h_{S}$ and $G_{S}$ are the structure-based representation for head to similar that studied in translation-based fashions along with transformation of $E$ (Wang, Ji, et al., 2020). In other case the based presentation of $h_{e}$ and $G_{e}$ descriptions based totally on the definition of head and tail constructed with business descriptions. we will propose codes to construct this type of presentation within the next phase(Sun et al., 2021).

\section{Approach}

if you want to observe each definition of 3-dimensional records and the definition of a commercial enterprise and a good way to address a meaningless scenario, we endorse the forms of commercial enterprise displays, structure-based presentation and definition-primarily based displays (Tao et al., 2021). We examine the totally display the carry out three intense as tons as knowledge graph in statistics, whilst descriptive reports do better with capturing textual facts in enterprise descriptions. We examine two commercial enterprise shows concurrently within the equal vector non-stop method, but do not force the shows to be blended to don't forget the potential to better represent (Tao et al., 2021). The description embodied knowledge representing learning is function is then described as

$$
E=E_{D}+E_{G}
$$

Where the $E_{D}$ is indicating the energy function of the structure-based representation strength function, which stocks the same path as of Eq1, while $E_{G}$ is representing the energy function of description-based representation(Nicholson \& Greene, 2020). And it can be described in an expansion of methods. The $E_{D}$ getting to know system with $E_{G}$ as follows:

$$
E_{G}=E_{G G}+E_{D G}+E_{G S}
$$

The Eq 2 is indicating $E_{G G}$ with $\left\|h_{G}+r-t_{G}\right\|$ where to integrate the

The pinnacle and tail are shows primarily based on definition. We also have $E_{D G}=\left\|h_{G}+r-t_{s}\right\|$ and $E_{G S}=\left\|h_{s}+r-t_{D}\right\|$, in which one of the $\mathrm{h}$ or $\mathrm{t}$ makes use of a descriptive representation of determined techniques and its totally illustrated the alternative makes use of a structure representation. The energy feature will show varieties of business presentations within the same vector with associated shows assigned to all 4 kind of energy features, in order to have the same advertising among the two kinds of displays. on this paper, we advise encoders to create descriptive presentations within the following paragraphs (Tang et al., 2021). First, we endorse the wallet encoder for commercial enterprise constructing, then we propose the convolutional neural network codec to higher recognize the textual records (X. Zhang et al., 2021).

In case of brief description, it produces a set of broad keywords that are to capture the basic identical ideas. The relationship cannot be detected by structural information by CBOW, so therefore the top identical keywords in each business as TF-IDF with including measure key (Gharaee et al., 2021). We imply the keywords with commands

$$
E_{d}=x_{1}+x_{2} \ldots \ldots+x_{n}
$$

Where $x_{n}$ is the insertion of the word th which is part of the keyword business e, and Ed will be used to reduce ED. The figure is interpreting the $\mathrm{CBOW}$ encoder by

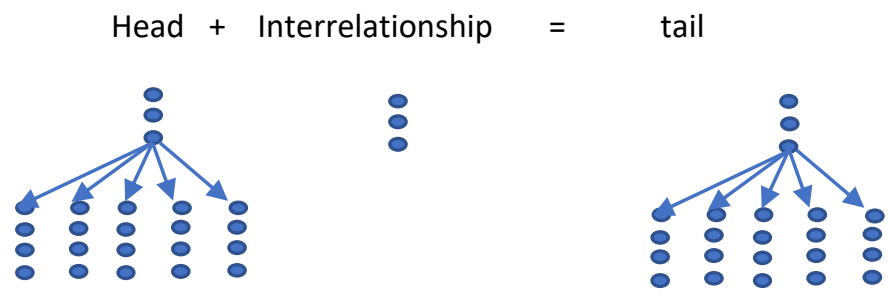

Figure 2: Encoder of CBOW 


\section{Learning of Knowledge Graphs with Entity Descriptions}

Encoder Convolutional neural network is an efficient model widely used on image and proven to be effective on some natural language processing tasks such as part-of-speech tagging, chunking, named entity recognition and semantic role in the model. Since CBOW has the shortage of ignoring the information of word orders and is easy to be influenced by the quality of keywords extraction(Ke et al., 2021; Riba et al., 2021).

\section{Initialization of the descriptive model}

In this structure the $\mathrm{CBOW}$ and CNN connectors take blank texts like embedding and business embedding as output to minimize imitation of the homework mentioned above. In order to maximize efficiency, we also use CNN's multimedia version to read presentations(Lee et al., 2021). The general backward rotation using a stochastic gradient descent (SGD) with the Rear propagation will be prevented if it meets a zero finish or the current value of the feature was not considered in the compilation during the forward broadcast(Lee et al., 2021).

\section{Dataset experiments}

In the Settings Datasets on this paper, we undertake a dataset extracted from a normal large-scale kingdom Freebase to assess with KDAL (CBOW) model and classify the affirm that every entity should have description for the illustration mastering. We categories and put off 56 entities from FB15K that have shorter than 5 phrases after preprocessing or maybe have no descriptions, and cast off all triples containing the ones entities. The average wide variety of words in descriptions is 76 after preprocessing, and the longest description includes 453 words. The remaining education set has 483,960 with 1482 relationship and identical test based on 64,904. Randomly we identify the 3554 new entities from the candidates that in experiments. With 6 identical shapes the dataset is highlighted.

Table 1. Dataset

\begin{tabular}{l|l|l|l|l|l}
\hline Dataset & $\mathrm{R}$ & Ent & Training & Validity & Results \\
\hline FEB15KA & 1482 & 14578 & 584576 & 483960 & 64904 \\
\hline FBA20KE & 194561 & 64904 & 208542 & 126542 & 213 \\
\hline
\end{tabular}

We use implanted structure of CNN by educate the ones fashions relation measurement within the CBOW Encoder, we try a exceptional variety of top $\mathrm{N}$ keywords and select the pinnacle 20 keywords to create enterprise bedding to do our exceptional. Also, we try to additionally set the width with 75 graphs of the datasets (Table 1).

Table 2. Results

\begin{tabular}{l|l|l|l|l}
\hline \multicolumn{1}{c|}{ Metrics of datasets } & \multicolumn{1}{|c|}{$\begin{array}{c}\text { Ranking means with } \\
\text { Raw }\end{array}$} & Filter & Raw & Filter \\
\hline Etrans & 345 & 320 & 54.7 & 57.9 \\
\hline KDAL(CBOW) & 328 & 120 & 25.9 & 32.9 \\
\hline DKLR(CNN) & 130 & 109 & 34.7 & 54.9 \\
\hline
\end{tabular}

Table 2 is indicated results of ranking mean of raw and filter with KDAL (CBOW) and DKLR (CNN), where the significant outperforms CBOW and mean rank.

Table 3. Relationship prediction

\begin{tabular}{l|l|l|l|l}
\hline Datasets & Raw & Filter & Raw & Filter \\
\hline Etrans & 3.25 & 3.22 & 54.3 & 60.9 \\
\hline KDAL(CBOW) & 3.21 & 3.19 & 45.6 & 67.4 \\
\hline DKLR(CNN) & 3.10 & 2.54 & 34.6 & 87.7 \\
\hline
\end{tabular}

Table 3 is indicated that CNN representation. We randomly divided it right into a schooling set and a test set, the training set by the above tables(J. Zhang et al., 2021). We use Logistic Regression as classifier and to make some other comparison, we use the conventional text feature (BOW) function as a base. Subsequent trying out. 


\section{Learning of Knowledge Graphs with Entity Descriptions}

All the descriptive older models based on structural presentation cannot cope with this situation because they are not able to represent businesses outside the KG. However, the KDAL(CBOW) model is naturally aware of this situation(Lu et al., 2021).

We use DKLR(CNN) to emulate the trivial situation that all businesses that are KG businesses can be trained through training, and view $\mathrm{CBOW}$ as our foundation as all existing models that use structural-based presentations are not able to represent new businesses. It shows that even in the simplest case, the KDAL(CBOW) model can still obtain positive results compared to the completion of the information graph(B. Zhang et al., 2021). However, there are still discrepancies between the two presentations that will weaken performance, compared to the parallel models of CNN and CBOW that use descriptive representation of all businesses.

It is effective considers enterprise definitions by way of mastering illustration, while there is specific information such as courting information or business types that can be introduced to our version.

\section{INFERENCE}

Work in this paper, we suggest a $\operatorname{KDAL}(\mathrm{CBOW})$ version to symbolize the reading of graphs of enterprise definitions. We're trying out two encoders that include a continuous phrase bag and a deep convolutional neural community to generate semantics for business definitions. Inside the checks, we tested our model in two obligations that included know-how graph of completion and commercial enterprise classification. Test effects show that our model achieves higher overall performance than other foundations in both operations specifically in the insignificant context, demonstrating the potential for constructive displays from enterprise descriptions. we will discover the following studies hints inside the future: (1) The KDAL(CBOW) version most effective considers enterprise definitions by way of mastering illustration, while there are 2664 specific information such as courting information or business types that can be introduced to our version(Romanov, 2021). We can be able to enhance with that wealthy expertise in the future. (2) We verify the validity of the definition-based submissions handiest through TransE, and it is not tough to similarly evaluate the most superior extension fashions.

\section{REFERENCES}

1) Bai, L., Yu, W., Chen, M., \& Ma, X. (2021). Multi-hop reasoning over paths in temporal knowledge graphs using reinforcement learning. Applied Soft Computing, 103, 107144. https://doi.org/https://doi.org/10.1016/j.asoc.2021.107144

2) Bellomarini, L., Fayzrakhmanov, R. R., Gottlob, G., Kravchenko, A., Laurenza, E., Nenov, Y., Reissfelder, S., Sallinger, E., Sherkhonov, E., Vahdati, S., \& Wu, L. (2022). Data science with Vadalog: Knowledge Graphs with machine learning and reasoning in practice. Future Generation Computer Systems, 129, 407-422. https://doi.org/https://doi.org/10.1016/j.future.2021.10.021

3) Gharaee, Z., Kowshik, S., Stromann, O., \& Felsberg, M. (2021). Graph representation learning for road type classification. Pattern Recognition, 120, 108174. https://doi.org/https://doi.org/10.1016/j.patcog.2021.108174

4) Huang, Y., Sun, H., Xu, K., Lu, S., Wang, T., \& Zhang, X. (2021). CoRelatE: Learning the correlation in multi-fold relations for knowledge graph embedding. Knowledge-Based Systems, 213, 106601. https://doi.org/https://doi.org/10.1016/j.knosys.2020.106601

5) Jia, J., Zhang, Y., \& Saad, M. (2022). An approach to capturing and reusing tacit design knowledge using relational learning for knowledge graphs. Advanced Engineering Informatics, 51, 101505. https://doi.org/https://doi.org/10.1016/j.aei.2021.101505

6) Ke, J., Feng, S., Zhu, Z., Yang, H., \& Ye, J. (2021). Joint predictions of multi-modal ride-hailing demands: A deep multi-task multi-graph learning-based approach. Transportation Research Part C: Emerging Technologies, $127,103063$. https://doi.org/https://doi.org/10.1016/j.trc.2021.103063

7) Ko, H., Witherell, P., Lu, Y., Kim, S., \& Rosen, D. W. (2021). Machine learning and knowledge graph based design rule construction for additive manufacturing. Additive Manufacturing, 37, 101620.

https://doi.org/https://doi.org/10.1016/j.addma.2020.101620

8) Lampropoulos, G., Keramopoulos, E., \& Diamantaras, K. (2020). Enhancing the functionality of augmented reality using deep learning, semantic web and knowledge graphs: A review. Visual Informatics, 4(1), 32-42. https://doi.org/https://doi.org/10.1016/j.visinf.2020.01.001

9) Lee, W.-K., Shin, W.-C., Jagvaral, B., Roh, J.-S., Kim, M.-S., Lee, M.-H., Park, H.-K., \& Park, Y.-T. (2021). A path-based relation networks model for knowledge graph completion. Expert Systems with Applications, $182,115273$. https://doi.org/https://doi.org/10.1016/j.eswa.2021.115273 


\section{Learning of Knowledge Graphs with Entity Descriptions}

10) Li, C., Peng, X., Niu, Y., Zhang, S., Peng, H., Zhou, C., \& Li, J. (2021). Learning graph attention-aware knowledge graph embedding. Neurocomputing, 461, 516-529. https://doi.org/https://doi.org/10.1016/j.neucom.2021.01.139

11) Li, J., Horiguchi, Y., \& Sawaragi, T. (2022). Counterfactual inference to predict causal knowledge graph for relational transfer learning by assimilating expert knowledge --Relational feature transfer learning algorithm. Advanced Engineering Informatics, 51, 101516. https://doi.org/https://doi.org/10.1016/j.aei.2021.101516

12) Li, Q., Li, L., Zhong, J., \& Huang, L. F. (2020). Real-time sepsis severity prediction on knowledge graph deep learning networks for the intensive care unit. Journal of Visual Communication and Image Representation, 72, 102901. https://doi.org/https://doi.org/10.1016/j.jvcir.2020.102901

13) Li, Z., Liu, H., Zhang, Z., Liu, T., \& Shu, J. (2021). Recalibration convolutional networks for learning interaction knowledge graph embedding. Neurocomputing, 427, 118-130. https://doi.org/https://doi.org/10.1016/j.neucom.2020.07.137

14) Lu, Y., Chen, Y., Zhao, D., \& Li, D. (2021). MGRL: Graph neural network based inference in a Markov network with reinforcement learning for visual navigation. Neurocomputing, 421, 140-150.

https://doi.org/https://doi.org/10.1016/j.neucom.2020.07.091

15) Nicholson, D. N., \& Greene, C. S. (2020). Constructing knowledge graphs and their biomedical applications. Computational and Structural Biotechnology Journal, 18, 1414-1428. https://doi.org/https://doi.org/10.1016/j.csbj.2020.05.017

16) Pham, T., Tao, X., Zhang, J., Yong, J., Li, Y., \& Xie, H. (2022). Graph-based multi-label disease prediction model learning from medical data and domain knowledge. Knowledge-Based Systems, 235, 107662.

https://doi.org/https://doi.org/10.1016/j.knosys.2021.107662

17) Riba, P., Fischer, A., Lladós, J., \& Fornés, A. (2021). Learning graph edit distance by graph neural networks. Pattern Recognition, 120, 108132. https://doi.org/https://doi.org/10.1016/j.patcog.2021.108132

18) Romanov, V. N. (2021). Deep-freeze graph training for latent learning. Computational Materials Science, $199,110757$. https://doi.org/https://doi.org/10.1016/j.commatsci.2021.110757

19) Shao, T., Li, X., Zhao, X., Xu, H., \& Xiao, W. (2021). DSKRL: A dissimilarity-support-aware knowledge representation learning framework on noisy knowledge graph. Neurocomputing, 461, 608-617. https://doi.org/https://doi.org/10.1016/j.neucom.2021.02.099

20) Shi, D., Wang, T., Xing, H., \& Xu, H. (2020). A learning path recommendation model based on a multidimensional knowledge graph framework for e-learning. Knowledge-Based Systems, 195, 105618.

https://doi.org/https://doi.org/10.1016/j.knosys.2020.105618

21) Sun, D., Li, D., Ding, Z., Zhang, X., \& Tang, J. (2021). Dual-decoder graph autoencoder for unsupervised graph representation learning. Knowledge-Based Systems, 234, 107564. https://doi.org/https://doi.org/10.1016/j.knosys.2021.107564

22) Tang, H., Ma, G., He, L., Huang, H., \& Zhan, L. (2021). CommPOOL: An interpretable graph pooling framework for hierarchical graph representation learning. Neural Networks, 143, 669-677. https://doi.org/https://doi.org/10.1016/j.neunet.2021.07.028

23) Tao, S., Qiu, R., Ping, Y., \& Ma, H. (2021). Multi-modal Knowledge-aware Reinforcement Learning Network for Explainable Recommendation. Knowledge-Based Systems, 227, 107217. https://doi.org/https://doi.org/10.1016/j.knosys.2021.107217

24) Tiddi, I., \& Schlobach, S. (2022). Knowledge graphs as tools for explainable machine learning: A survey. Artificial Intelligence, 302, 103627. https://doi.org/https://doi.org/10.1016/j.artint.2021.103627

25) Tiwari, P., Zhu, H., \& Pandey, H. M. (2021). DAPath: Distance-aware knowledge graph reasoning based on deep reinforcement learning. Neural Networks, 135, 1-12. https://doi.org/https://doi.org/10.1016/j.neunet.2020.11.012

26) Wang, Q., Hao, Y., \& Cao, J. (2020). ADRL: An attention-based deep reinforcement learning framework for knowledge graph reasoning. Knowledge-Based Systems, 197, 105910. https://doi.org/https://doi.org/10.1016/j.knosys.2020.105910

27) Wang, Q., Ji, Y., Hao, Y., \& Cao, J. (2020). GRL: Knowledge graph completion with GAN-based reinforcement learning. Knowledge-Based Systems, 209, 106421. https://doi.org/https://doi.org/10.1016/j.knosys.2020.106421

28) Yu, C., Wang, F., Liu, Y.-H., \& An, L. (2021). Research on knowledge graph alignment model based on deep learning. Expert Systems with Applications, 186, 115768. https://doi.org/https://doi.org/10.1016/j.eswa.2021.115768

29) Zhang, B., Leung, K.-C., Li, X., \& Ye, Y. (2021). Learn to abstract via concept graph for weakly-supervised few-shot learning. Pattern Recognition, 117, 107946. https://doi.org/https://doi.org/10.1016/j.patcog.2021.107946

30) Zhang, J., Chen, B., Zhang, L., Ke, X., \& Ding, H. (2021). Neural, symbolic and neural-symbolic reasoning on knowledge graphs. Al Open, 2, 14-35. https://doi.org/https://doi.org/10.1016/j.aiopen.2021.03.001 


\section{Learning of Knowledge Graphs with Entity Descriptions}

31) Zhang, X., Yang, Y., Zhai, D., Li, T., Chu, J., \& Wang, H. (2021). Local2Global: Unsupervised multi-view deep graph representation learning with Nearest Neighbor Constraint. Knowledge-Based Systems, 231, 107439. https://doi.org/https://doi.org/10.1016/j.knosys.2021.107439

32) Zhao, Y., Li, Z., Deng, W., Xie, R., \& Li, Q. (2021). Learning entity type structured embeddings with trustworthiness on noisy knowledge graphs. Knowledge-Based Systems, 215, 106630.

https://doi.org/https://doi.org/10.1016/j.knosys.2020.106630

33) Zhao, Y., Zhang, A., Feng, H., Li, Q., Gallinari, P., \& Ren, F. (2020). Knowledge graph entity typing via learning connecting embeddings. Knowledge-Based Systems, 196, 105808. https://doi.org/https://doi.org/10.1016/j.knosys.2020.105808

There is an Open Access article, distributed under the term of the Creative Commons Attribution - Non Commercial 4.0 International (CC BY-NC 4.0)

(https://creativecommons.org/licenses/by-nc/4.0/), which permits remixing, adapting and building upon the work for non-commercial use, provided the original work is properly cited. 